\title{
ERFOLGSFAKTOREN VON LEHRSTELLENMARKETING IN DER DUALEN BERUFSBILDUNG: DAS BEISPIEL SCHWEIZ
}

\author{
FRITZ SAGER
}

\begin{abstract}
SUCCESS FACTORS OF APPRENTICESHIP MARKETING IN A SYSTEM OF DUAL VOCATIONAL TRAINING: THE CASE OF SWITZERLAND - The dual system of vocational training, utilizing both company training and vocational school, is generally acknowledged to be a successful model, but a decreasing number of trainee posts in Switzerland poses a crisis for the approach. One strategy for overcoming the problem involves offering incentives for companies to create new trainee posts. The present study explores the necessary conditions for successfully influencing the number of trainee posts through apprenticeship marketing. A comparision of qualitative case studies of six marketing projects demonstrates that while context, in the sense of basic structural conditions and political sensibility for the problematic, plays a role in the success of a project, what is central is above all the form of the trainee-post marketing project itself. This has to do with the behaviour of the actors involved as well as the choice of the mode of governance and project organization. The latter has to do with making available requisite technical and personal resources, coordination on the part of the project direction, and giving those responsible the necessary room to act in implementing the project.
\end{abstract}

\begin{abstract}
Zusammenfassung - ERFOLGSFAKTOREN VON LEHRSTELLENMARKETING IN DER DUALEN BERUFSBILDUNG: DAS BEISPIEL SCHWEIZ - Das duale Berufsbildungssystem, das die praktische Ausbildung in einem Betrieb mit theoretischem Unterricht in der Berufsschule verbindet, wird generell als erfolgreiches Modell für die Berufsbildung bezeichnet, steckt aber angesichts einer abnehmenden Anzahl von Ausbildungsplätzen in der Schweiz aktuell in einer Krise. Eine Strategie, um diesem Problem zu begegnen, ist die Bereitstellung von Anreizen für Betriebe, neue Lehrstellen zu schaffen. Der vorliegende Beitrag fragt nach den Bedingungen für eine erfolgreiche Beeinflussung des Lehrstellenangebotes durch Lehrstellenmarketing. Ein qualitativer Fallstudienvergleich von sechs Marketingprojekten führt zur Erkenntnis, dass der Kontext im Sinne struktureller Rahmenbedingungen und politischer Sensibilisierung für die Lehrstellenproblematik eine Rolle für den Erfolg eines Projekts spielt, aber vor allem die Form des Lehrstellenmarketingprojekts selber zentral ist. Dies betrifft das Auftreten der beteiligten Akteure ebenso wie die gewählte Art der politischen Steuerung und der Organisation eines Projekts. Letztere bezieht sich auf die Bereitstellung der notwendigen fachlichen und personellen Ressourcen, die Koordinationsfähigkeit der Projektleitung und die Gewährleistung der notwendigen Handlungsspielräume der Projektverantwortlichen bei der Umsetzung.
\end{abstract}

Résumé - FACTEURS DE SUCCÈS DE LA MERCATIQUE DES PLACES D'APPRENTISSAGE DANS LA FORMATION PROFESSIONNELLE BINAIRE: L'EXEMPLE DE LA SUISSE - le système de formation professionelle binaire avec une formation en entreprise et une école de formation professionelle est généralement qualifié de modèle réussi pour la formation professionnelle, il se trouve toutefois en crise vu le 
nombre actuellement décroissant de lieux de formation en Suisse. Une stratégie pour faire face à ce problème est de mettre à disposition des entreprises des incitations à créer de nouvelles places d'apprentissage. La présente contribution interroge les conditions influençant avec succès l'offre de places d'apprentissage par le biais d'une mercatique des places d'apprentissage. Une comparaison qualitative d'études de cas de six projets de mercatique conduit à la constatation que le contexte, dans le sens de conditions générales structurelles et de sensibilisation politique à la problématique des places d'apprentissage, joue un rôle pour le succès d'un projet, mais que c'est surtout la forme du projet de mercatique des places d'apprentissage elle-même qui est centrale. Cela concerne aussi bien le comportement des acteurs qui y participent que le choix qui sera fait du mode de governance politique et l'organisation d'un projet. Cette dernière se réfère à la mise à disposition de ressources professionnelles et humaines nécessaires, à la capacité de coordination de la gestion du projet et à la garantie de marges de manœuvre nécessaires des responsables du projet lors de sa mise en œuvre.

\begin{abstract}
Resumen - LOS FACTORES DETERMINANTES DEL ÉXITO DE LAS ESTRATEGIAS DE MARKETING PARA PROMOVER LA FORMACIÓN PROFESIONAL DUAL: EL EJEMPLO DE SUIZA - El sistema de formación profesional dual, o sea de aprendizaje práctico en la empresa y teórico en la escuela técnica, es considerado un modelo de éxito. Sin embargo, en Suiza este sistema está atravesando una crisis, debida a la disminución de plazas disponibles para la formación profesional. Una de las estrategias para enfrentar el problema es la de ofrecer incentivos para que las empresas creen nuevos puestos de aprendizaje. Este trabajo se centra en las condiciones que debe reunir un marketing de formación profesional para poder influenciar positivamente la oferta de estos puestos de aprendizaje. De la comparación cualitativa de seis proyectos de marketing concretos, se puede deducir que el contexto de condiciones marco estructurales y de concienciación política sobre el problema de los puestos de aprendizaje juega un cierto papel para el éxito de un proyecto, pero que ante todo es fundamental la forma en la que se realice el proyecto mismo. Esto atañe al modo de presentarse de los actores involucrados al igual que a la forma que se ha elegido para el control político y la organización de un proyecto; esta última está relacionada con la disponibilidad de recursos técnicos y personales, la capacidad de coordinación de la dirección del proyecto y la garantía que se de a los responsables del proyecto para que puedan llevarlo a la práctica con la libertad de acción necesaria.
\end{abstract}

Резюме - ФАКТОРЫ, СОСТАВЛЯЮЩИЕ УСПЕХ В МАРКЕТИНГЕ РАБОЧИХ МЕСТ ДЛЯ СТАЖЕРОВ В СИСТЕМЕ ДУАЛЬНОЙ ПРОФЕССИОНАЛЬНОЙ ПОДГОТОВКИ: СИТУАЦИЯ В ШВЕЙЦАРИИ - Дуальная система профессиональной подготовки, использующаяся в качестве тренинга как в компаниях, так и в профессиональных училищах, в общем признается успешной моделью, но уменьшающееся количество рабочих мест для стажеров в Швейцарии ставит такой подход в кризисное положение. Одна из стратегий для разрешения этой проблемы включает создание такого стимула, чтобы компании предоставляли новые рабочие места для стажеров. В данном исследовании рассматриваются необходимые условия для оказания позитивного влияния на количество рабочих мест для стажеров через их маркетинг. Качественное исследование примеров шести маркетинговых проектов показывает, что пока контекст в смысле базовых структурных условий и политической остроты проблематики играет роль в успешности проведения проекта, центральным вопросом, прежде всего, остается форма самого проекта маркетинга рабочих мест для стажеров. Это имеет отн- 
ошение к поведению участников-акторов, а также к выбору политического контроля и организации проекта. Последнее связано с обеспечением доступности необходимых технических и человеческих ресурсов, с координацией со стороны управления проектом и с предоставлением ответственным лицам необходимого пространства для действий и осуществления самого проекта.

Wie Deutschland und Österreich organisiert die Schweiz die Berufsbildung im so genannten dualen System. Das duale System zeichnet sich dadurch aus, dass die Berufsbildung primär in der Berufslehre in einem Betrieb erfolgt, begleitet vom Besuch einer Berufsfachschule (duales System) oder begleitet von Berufsfachschule und überbetrieblichen Kursen (triales System). Das duale System wird generell als erfolgreiches Modell für die Berufsbildung bezeichnet (BIBB 2004; Labarca 1998; Lee 1994; Müller und Shavit 1998) und dient daher auch als Vorbild für Reformen des Berufsbildungssystems in verschiedenen europäischen Ländern (Borham 2002).

Gleichwohl muss angesichts einer abnehmenden Anzahl von Ausbildungsplätzen und einem Zuwachs bei den Lehrabbrüchen aktuell von einer Krise des dualen Systems gesprochen werden (Deißinger und Hellwig 2004), die die jeweiligen Regierungen dazu anhält, Maßnahmen und Strategien zur Aufrechterhaltung des Systems zu ergreifen. Eine solche Strategie ist die Bereitstellung von Anreizen für Betriebe, neue Lehrstellen zu schaffen. Dieses so genannte Lehrstellenmarketing ist Gegenstand des vorliegenden Artikels, der nach den Bedingungen für eine erfolgreiche Beeinflussung des Lehrstellenangebotes fragt. Die berichteten Ergebnisse basieren auf der systematischen Evaluation der Lehrstellenmarketingmaßnahmen im Rahmen eines bundesstaatlichen Programms zur Lehrstellenförderung in der Schweiz (Sager, Schläpfer und Giraud 2004).

Der Artikel ist wie folgt aufgebaut: In einem ersten Abschnitt wird der Begriff des Lehrstellenmarketings eingeführt und entsprechende Policy-Instrumente identifiziert. Im zweiten Schritt wird auf den politischen Kontext von Lehrstellenmarketing in der Schweiz eingegangen. Dies umfasst zum einen die Entwicklung der Lehrstellensituation und zum anderen die Besonderheiten des schweizerischen Berufsbildungssystems. Vor diesem Hintergrund werden in einem dritten Schritt die Ergebnisse der Evaluationsstudie des schweizerischen Lehrstellenmarketings präsentiert. Im letzten Kapitel werden die wichtigsten Erkenntnisse zusammengefasst und Schlussfolgerungen für die Sicherung der Zukunft des dualen Systems gezogen.

\section{Definition von Lehrstellenmarketing}

Der Berufsbildungsmarkt ist ein spezieller Markt, in dem die Nachfrage primär vom Angebot bestimmt wird (Angebotsmarkt). So sind die Betriebe als Anbieter von Lehrstellen die entscheidenden Akteure im Berufsbildungsmarkt. 
Grundsätzlich stellt sich damit die Frage, was der Staat auf einem solch angebotsorientierten Markt überhaupt ausrichten kann (Wolter, Nagel-Drdla und Waibel 2001). Lehrstellenmarketing bezeichnet entsprechende politische Anstrengungen, das Lehrstellenangebot zu vergrößern.

Der Begriff Lehrstellenmarketing kennt keine allgemeingültige Definition und wird entsprechend für eine Vielzahl unterschiedlicher Maßnahmen verwendet. So bezeichnen beispielsweise Gertsch und Hotz (1999: 4) alle Maßnahmen, die direkt auf die Erhaltung und Ausweitung des Lehrstellenangebotes ausgerichtet sind, als Marketingmaßnahmen. Schucan (2002: 9) plädiert aufgrund der konjunkturell und strukturell unterschiedlichen Entwicklung am Lehrstellenmarkt (es gibt gleichzeitig Lehrstellen- und Nachwuchsmangel) für eine Erweiterung des Konzepts des Lehrstellenmarketings zu einem umfassenden Berufsbildungsmarketing. Alle Maßnahmen, die dazu beitragen, dass der Markt der beruflichen Grundbildung gut funktioniert, gehören demnach zum Berufsbildungsmarketing. Ziel ist es, dass die Jugendlichen genügend und geeignete Ausbildungsplätze finden, die Lehrbetriebe genügend und geeignete Lernende und Nachwuchs erhalten und dass der Volkswirtschaft genügend und geeignete Fachkräfte zur Verfügung gestellt werden.

Im Folgenden werden Maßnahmen als Lehrstellenmarketing bezeichnet, die entweder eine gezielte Erhöhung des Angebotes betrieblicher Ausbildungsplätze anstreben oder die die Nachfrage bei den Jugendlichen in Richtung der Berufssektoren mit offenen Lehrstellen zu beeinflussen versuchen. Eine Analyse der bestehenden Maßnahmen führt zu einer Kategorisierung von Lehrstellenmarketing, bei der vier Typen mit jeweils zugehörigen Instrumenten unterschieden werden (Table 1).

\section{Der politische Kontext von Lehrstellenmarketing}

\section{Entwicklung der Lehrstellensituation}

Seit Mitte der Achtzigerjahre hat in der Schweiz die Zahl der abgeschlossenen Lehrverträge kontinuierlich abgenommen. Sowohl die Zahl der Unternehmen, welche Ausbildungsplätze anbieten, wie auch die Zahl der zur Verfügung gestellten Ausbildungsplätze gingen tendenziell zurück. Ab 1995 konnte dieser Trend gestoppt werden, und die Zahl der Lehrstellen und sonstigen Ausbildungsplätze stieg wieder an (Bundesrat 2000: 5694). Utiger (2003: 12) zeigt, dass zwischen der Entwicklung der Gesamtbeschäftigung und der Anzahl der Lehrstellen wenig Übereinstimmung besteht. So wurden in der Schweiz zwischen 1985 und 20013.482 oder 15,1 Prozent aller Lehrstellen abgebaut, während in der gleichen Zeit die Gesamtbeschäftigung um 192.400 Vollzeitäquivalente oder 6,5 Prozent zunahm. Diese Entwicklung ist vor allem für den immer wichtiger werdenden Dienstleistungssektor charakteristisch. Der Arbeitsmarkt unterliegt seit den Neunzigerjahren tief greifenden strukturellen Veränderungen, die durch die konjunkturelle Entwicklung noch 
Table 1. Arten und Instrumente des Lehrstellenmarketings

\begin{tabular}{ll}
\hline Art des Lehrstellenmarketings & Instrumente \\
\hline Information & Standaktionen an Ausstellungen \\
& Organisation von spezifischen \\
& Berufsbildungsveranstaltungen \\
Werbekampagnen & Durchführen von Kursen \\
& Mailings \\
& Flyer und Inserate \\
& Publikationen und Informationsbroschüren \\
& Homepages auf dem Internet \\
& Ausbildungsforen \\
& Neue Lehrmodelle \\
& Ausbildungsverbund \\
& Vereinigung von Berufsbildungsverantwortlichen der \\
Institutionelle und & betrieblichen Ausbildung \\
Komprastrukturmaßnahmen & Basislehrgänge \\
& Staatliche Kostenbeteiligungen \\
Finanzielle Anreize & Netzwerkaufbau \\
Intervention & Betriebsbesuche zwecks Akquisition und Beratung \\
& Coaching von Betrieben und Jugendlichen \\
& Lehrstellenförderer \\
& Akquisition mittels Mailings und Telefon
\end{tabular}

verstärkt und beschleunigt wurden (Gertsch und Hotz 1999: 3). So verlangt beispielsweise die dynamische Entwicklung der neuen Kommunikations- und Informationstechnologien Änderungen bei den Anforderungen an die Berufe und sogar an ganze Berufsfelder. Damit ist auch das duale Ausbildungssystem vor neue Herausforderungen gestellt (Bundesrat 2000: 5693). Der zunehmende Spezialisierungsgrad von Kleinbetrieben in den zukunftsträchtigen HightechBereichen (z.B. Software-Branche) und in anspruchsvollen Dienstleistungssegmenten (z.B. Beratungsfirmen) schränkt das Angebot von Lehrstellen ein. Nur wenige dieser Kleinbetriebe sind in der Lage, das ganze erforderliche Ausbildungsspektrum abzudecken und Lehrstellen anzubieten (Bundesrat 2000: 5694; Wolter, Mülemann und Schweri 2003). Neben den strukturellen Gründen werden aber auch demographische Gründe für die negative Entwicklung am Lehrstellenmarkt geltend gemacht. Seit Mitte der Neunzigerjahre steigt die Zahl der Schulabgängerinnen und Schulabgänger stetig an, was zu einer größeren Lehrstellennachfrage und damit zu einer Verschärfung der Problematik auf dem Lehrstellenmarkt geführt hat.

\section{Besonderheiten des schweizerischen Berufsbildungssystems}

Das schweizerische Berufsbildungssystem ist durch zwei politisch-institutionelle Besonderheiten gekennzeichnet. Zum einen zeichnet es sich durch 
Subsidiarität aus, womit eine ausgeprägte Arbeitsteilung zwischen staatlichen und wirtschaftlichen Akteuren gemeint ist. Zum anderen ist das schweizerische politische System ausgesprochen föderalistisch, was insbesondere auf den Bereich der Bildung zutrifft (Freitag und Bühlmann 2003). Diese zwei Merkmale führen zum einen zu regionalen Unterschieden in der Berufsbildung, insbesondere zwischen der deutschen und der lateinischen Schweiz, die durch die unterschiedlichen Traditionen der einzelnen Gliedstaaten der Schweiz, der Kantone, erklärt werden können. Zum anderen führt das fragmentierte Berufsbildungssystem auch zu einer Vielzahl unterschiedlicher Ansätze, was zu einem gewissen Grad innovationsfördernd wirkt.

\section{Sprachregionale Unterschiede}

Vor allem in der deutschen Schweiz ist die duale Berufsbildung mit Betriebslehre die dominante Form. Einige Berufe können auch alternativ in einer Betriebslehre oder in einer Fachschule erlernt werden. In der französischsprachigen Westschweiz und im italienischsprachigen Tessin ist die Berufsbildung jedoch strukturell anders aufgebaut und von den romanischen Nachbarländern mit ihrer schulisch ausgerichteten Berufsausbildung beeinflusst. So sind berufliche Vollzeitschulen in der lateinischen Schweiz viel häufiger vertreten als in der Deutschschweiz (DBK 2004). Die Finanzierung der Berufsbildung ist demnach in der lateinischen Schweiz Sache des Staates, während in der deutschen Schweiz mehrheitlich die Wirtschaft die Kosten übernehmen muss und sich der Staat nur subsidiär daran beteiligt (Wolter und Schweri 2002, 2004). Die Probleme und Herausforderungen im Bereich der beruflichen Ausbildung sind somit in der deutschen und lateinischen Schweiz nicht identisch. In der deutschen Schweiz müssen einerseits wieder vermehrt Jugendliche für die gewerbliche Grundausbildung gewonnen werden und zum anderen sollen bestehende Ausbildungsplätze insbesondere im Hightech- und Dienstleistungsbereich erhalten und neue generiert werden. In der lateinischen Schweiz zielen die Marketingmaßnahmen in eine andere Richtung. Den stark schulbasierten Berufsausbildungen müssen Praktikumsmöglichkeiten in der Wirtschaft erschlossen werden. Um Berufsbildungskosten sparen zu können, sollen neue Betriebe, welche sich traditionell nur selten in der betrieblichen Grundausbildung engagieren, für die Ausbildung von Lernenden gewonnen werden. Mittels neuer Lehrmodelle, welche den Betrieben eine größtmögliche Auswahl und Flexibilität bei ihrer Ausbildung von Lernenden anbieten, soll das lateinische Berufsbildungssystem vermehrt in die Richtung des dualen und trialen Systems verändert werden.

Die strukturelle Verschiedenheit des Lehrstellenmarktes in den verschiedenen Regionen sowie die Dominanz der Anbieter müssen sowohl bei der Durchführung von Marketingmaßnahmen wie auch bei deren Evaluation berücksichtigt werden. 


\section{Institutionelle Fragmentierung als Innovationslabor}

Die einzigartige politisch-institutionelle Fragmentierung des schweizerischen Berufsbildungssystems ermöglicht eine Vielfalt von unterschiedlichen Lösungen und kann somit als Versuchslabor für Innovation bei der Entwicklung von politischen Lösungen für die Krise des dualen Systems gelten. Damit einher geht auch eine forschungstechnisch günstige Situation, die vor allem komparativen Analysen eine ideale Untersuchungsgesamtheit bietet. Im Bereich des Lehrstellenmarketings kann auf eine Vielzahl von Projekten zurückgegriffen werden, die sich sowohl hinsichtlich der Form des Lehrstellenmarketings als auch hinsichtlich ihrer Trägerschaft unterscheiden. Die hier präsentierte Untersuchung macht sich diese hervorragende Ausgangslage für vergleichende Forschung zunutze.

\section{Fragestellung Fallwahl und methodisches Vorgehen}

Das zentrale Erkenntnisinteresse der präsentierten Untersuchung liegt in der Beantwortung der Frage, welche Marketingmaßnahmen unter welchen Bedingungen erfolgreich sind. Um die Wirkungsweise und die Leistungsfähigkeit der verschiedenen Formen des Lehrstellenmarketings zu analysieren, wurde für jede identifizierte Form ein Fall im Rahmen einer qualitativen Einzelfallstudie vertieft untersucht. Die Fälle wurden anschließend mit einem vergleichenden Untersuchungsdesign einander gegenübergestellt.

Die Grundgesamtheit der zur Verfügung stehenden Fälle bestand aus 58 Lehrstellenmarketing-Projekten, die im Rahmen eines finanziellen Anreizprogramms des Bundes (dem so genannten Lehrstellenbeschluss 2, vgl. Bundesbeschluss 1999) gefördert wurden. Die Fallauswahl basierte auf zwei Kriterien. Die gewählten Projekte mussten zum einen die verschiedenen Typen von Lehrstellenmarketing abdecken. Zweitens mussten die Träger der gewählten Projekte die Vielfalt des schweizerischen Föderalismus sowie der Subsidiarität abdecken. Entsprechend wurden die folgenden Projekte untersucht:

\section{Deutschschweiz}

Ländliche Kantone mit Hauptprodukt Information: Projekt "Zusammenarbeit in der Berufsbildung Zentralschweiz - Teilprojekt Kommunikation"'

Das Zusammenarbeitsprojekt von sechs Kantonen bestand aus einem Masterprojekt und vier Teilprojekten, von denen sich das Teilprojekt "Kommunikation Berufsbildung Zentralschweiz" mit verschiedenen Informationsinstrumenten der Förderung der Berufsbildung widmete. Ab 2003 wurde Lehrstellenmarketing aufgrund der zunehmenden Lehrstellenknappheit im Dienstleistungssektor zum Schwerpunkt des Projekts. Das Projekt wird als Erfolg bezeichnet, da es einerseits seine operativen Ziele erreicht hat und 
zweitens zu nachhaltigen Zusammenarbeitsstrukturen beigetragen hat, die über das Projekt hinaus tragfähig sind.

\section{Kleiner urbaner Kanton mit Hauptprodukt Intervention: Projekt "Lehrstellenför- derer Basel-Landschaft",}

Der Lehrstellenförderer hat die Aufgabe, durch direkte Kontakte mit potenziellen Ausbildnern Lehrplätze zu generieren. Im untersuchten Projekt wurde der Schwerpunkt auf die Gründung und Betreuung eines Ausbildungsverbundes sowie auf die Förderung von Ausbildungsplätzen für schulisch schwache Jugendliche gelegt. Das Projekt wird als Erfolg bezeichnet, da mehr Ausbildungsplätze als erwartet neu geschaffen werden konnten.

\section{Großer Kanton mit Hauptprodukt Institution/Infrastrukturmaßnahmen: Projekt "Regionale Ausbildungsforen Zürich"}

Berufsbildungsforen (Ausbildungsforen, Lehrstellenforen) sind regionale Plattformen zur Behandlung von Fragen und Problemen an der Schnittstelle zwischen Volksschule und Berufsbildung und in weiteren Bereichen der Berufsbildung. Der Kanton Zürich betreibt über die Förderung der Foren ein indirektes Lehrstellenmarketing. Er unterstützt die Foren beim Aufbau und ihrer Tätigkeit, damit sie wiederum in ihrem Bezirk die Lehrstellensituation verbessern. Die Maßnahme des Kantons greift damit nicht direkt ein, sondern versucht über die Einrichtung neuer Strukturen Einfluss auf den Lehrstellenmarkt zu nehmen. Diese Form der indirekten Steuerung macht es schwierig, eine eigentliche Wirkungsabschätzung vorzunehmen. Das Projekt wird als teilweiser Erfolg bezeichnet, da die Foren unterschiedlich gut funktionieren.

\section{Französischsprachige Schweiz}

Kleiner Kanton mit Hauptprodukt Institution/Infrastrukturmaßnahme: Projekt "Partenariat école-industrie" in Neuchâtel

Das Projekt des Kantons Neuchâtel zielt auf den Wechsel von der schulischen Berufsbildung zum dualen System. Zu diesem Zweck wurden die Funktion des Lehrstellenförderers und eine nach-obligatorische Schulstruktur eingerichtet, die die angehenden Lernenden bei der Lehrstellensuche begleitet und in Zusammenarbeit mit den potenziellen Ausbildnern auf die Anforderungen in der Lehre vorbereitet. Das Projekt kann nicht als Erfolg bezeichnet werden, da weder der Lehrstellenförderer die angestrebte Anzahl neuer Ausbildungsplätze schaffen konnte, noch die Widerstände der potenziellen Ausbildner gegen das Konzept des dualen Systems ausgeräumt werden konnten.

Mittelgroßer Kanton mit Produkt "finanzielle Anreize": Projekt "Subvention des cours d'introduction" in Freiburg

Das einzige Projekt, das mit dem Typ "finanzielle Anreize" arbeitete, wurde im Kanton Freiburg durchgeführt. Der finanzielle Anreiz für Ausbildner, Lehrstellen zu schaffen, bestand im untersuchten Projekt darin, dass der 
Kanton die Hälfte der Kosten für die Einführungskurse für Erstjahreslernende übernahm, die sonst zulasten der Unternehmen gingen. Das Projekt wird als Misserfolg gewertet, da keine neu geschaffenen Lehrstellen nachgewiesen werden konnten, sondern primär bestehende Ausbildungsplätze subventioniert wurden. Nach Abschluss des Projektes wurde es nicht weitergeführt, da auch die zuständigen Personen in der Kantonsverwaltung gewechselt hatten.

\section{Berufsverband}

Beispiel für ein Projekt eines Schweizerischen Berufsverbands mit Hauptprodukt Information: Projekt "Lehrstellenmarketing Bäckerei-Konditorei- und Confiserie-Branche",

Das untersuchte Projekt hatte zwei zentrale Produkte: Zum einen wurde ein Ordner für die Verbandsmitglieder erstellt, der Informationen für den Kontakt mit Interessierten zusammenträgt. Zum anderen wurde ein Leitfaden für Schnupperlehrbetriebe erarbeitet, mit denen Lehrbetriebe die Eignung von Schnupperlehrlingen besser einschätzen können. Mit dieser Maßnahme sollte die Qualität der Lernenden, aber auch der Berufsbildungsverantwortlichen der betrieblichen Ausbildung verbessert werden. Das Projekt wurde als Erfolg gewertet, da es nicht nur alle operativen Ziele erreichte, sondern auch eine nachhaltige Wirkung entfaltete, die fortlaufend weiter kontrolliert wird.

Für die Untersuchung der Wirksamkeit von Lehrstellenmarketing wurde der Fokus nicht auf einzelne Maßnahmen, sondern auf die Kombination von Marketingmaßnahmen gelegt. Ebenfalls von Bedeutung war der soziostrukturelle und politische Kontext, in dem diese Maßnahmen zur Anwendung gelangten.

Für die empirische Arbeit wurde ein qualitatives Verfahren angewendet. In einem ersten Schritt wurden die bestehenden Dokumente zusammengetragen und gesichtet. Auf dieser Basis konnten ein Bild des Falles aufgebaut und Thesen für die nachfolgenden Interviews erarbeitet werden. Die halbstandardisierten Interviews mit den involvierten Akteuren wurden mit einem Gesprächsleitfaden geführt, der für jeden Fall spezifiziert wurde. Pro Fallstudie fanden durchschnittlich drei Interviews statt, wobei die Anzahl der Gesprächspartner bzw. Gesprächspartnerinnen abhängig vom einzelnen Fall variierte. Die Interviews stellten die zweite Grundlage für die Einzelfallanalyse dar.

Für den eigentlichen Fallstudienvergleich wurden die Erkenntnisse aus den einzelnen Fallstudien in die Gesamtheit der in der jeweiligen Fallstudie beobachteten Phänomene eingeordnet. Bei der Gegenüberstellung der Fälle gingen damit die Besonderheiten jedes einzelnen Falles nicht verloren und konnten gleichwohl Gemeinsamkeiten und Regelmäßigkeiten über den Einzelfall hinaus identifiziert werden. 


\section{Resultate aus dem Fallstudienvergleich}

Eine erste Erkenntnis aus den Fallstudien ist, dass keine eindeutige Aussage über den Erfolg einzelner Formen von Lehrstellenmarketing gemacht werden kann. Erfolg wurde in der Fallanalyse breit definiert: Als erfolgreich eingestuft wurden sowohl Projekte, in denen es gelang, die geplanten Maßnahmen in der vorgesehenen Art und Weise bereitzustellen, als auch Projekte, mit denen nachweislich Lehrstellen geschaffen werden konnten. Sowohl die Umsetzung als auch die erzielten Resultate wurden also als Indikatoren für den Erfolg eines Projektes gewertet.

Der Vergleich zeigt, dass der Erfolg oder Nicht-Erfolg nicht unmittelbar auf den gewählten Projekttyp zurückgeführt werden kann. Außerdem ermöglicht der Vergleich der untersuchten sechs Lehrstellenmarketing-Projekte die Identifikation von unterschiedlichen Faktoren, die unter jeweils spezifischen Bedingungen dazu beitragen, dass ein Projekt als Erfolg bezeichnet werden kann.

Diese Faktoren können auf vier Ebenen gruppiert werden. Erstens spielt der politische Kontext eine bedeutende Rolle für den Erfolg eines Projekts. Zweitens finden sich Aspekte des Prozessverlaufs und der beteiligten Akteure, die einen Einfluss auf die Realisierung und Entwicklung der Maßnahmen gehabt haben. Drittens lassen sich auf einer inhaltlichen Ebene Merkmale der gewählten Maßnahmen und Instrumente festmachen, die ebenfalls zum Projekterfolg beitragen. Viertens schließlich spielen organisatorische Faktoren eine wichtige Rolle in den untersuchten Lehrstellenmarketingprojekten.

\section{Kontextfaktoren}

Eine wichtige Rolle für den Erfolg eines Projekts spielt der Kontext, in welchem es stattfindet. Dies betrifft einerseits strukturelle Rahmenbedingungen und andererseits die politische Sensibilisierung für die Lehrstellenproblematik.

Einen ersten Faktor stellt die politische Ausgangslage dar. Es hat sich beim Zentralschweizer Lehrstellenmarketing gezeigt, dass die Konstellation der sechs Zentralschweizer Kantone eine günstige Voraussetzung für eine funktionierende Kooperation darstellte, indem die Kantone in einem klar definierten, geografischen Raum mit einem hohen Identifikationswert und grenzüberschreitenden Problemen liegen. Diese Konstellation führte dazu, dass die Zusammenarbeit im Berufsbildungssektor zu einem gewissen Grade auf der bereits funktionierenden und institutionalisierten Zusammenarbeit im Volksschulsektor aufbauen konnte. Von günstigen Rahmenbedingungen profitierten auch die Ausbildungsforen im Kanton Zürich. Hier konnte auf teilweise bereits bestehenden Netzwerken aufgebaut werden, die sich selbst zur Lösung der Lehrstellenproblematik konstituiert hatten. Auf der Gegenseite zeigten sich beim Neuenburger Projekt des 'Partenariat flexible' schwierige strukturelle Ausgangsbedingungen als problematisch für die Akzeptanz des Projekts. Zum einen haben die Berufsfachschulen traditionell ein größeres Gewicht als 
das kantonale Berufsbildungsamt, was eine breite Abstützung des Projekts in der Verwaltung erschwerte, zum anderen führte der angestrebte grundsätzliche Systemwechsel bei den wirtschaftlichen Akteuren zu einer großen Zurückhaltung. Generell ist hierbei anzumerken, dass der Umbau des Berufsbildungssystems in den eher schulisch ausgerichteten Westschweizer Kantonen einen größeren Wechsel bedeutet als in den stärker dual ausgerichteten Deutschschweizer Kantonen. Marketingmaßnahmen, die diesen Wechsel anzielen, haben hier schwerere Hürden auch institutioneller Art zu überwinden.

Ein zweiter Faktor besteht im politisch sensibilisierten Umfeld zum Zeitpunkt des Entscheids über und im Laufe der Umsetzung eines Projekts. Beim Fall des Zentralschweizer Lehrstellenmarketings zeigte sich, dass sowohl die Verunsicherung durch die Reform des Berufsbildungssystems als auch der politische Druck durch eine Volksinitiative auf Bundesebene - die 'Lehrstelleninitiative' der Sozialdemokratischen Partei der Schweiz, die die Bundeskompetenzen in der Berufsbildung verstärken wollte, aber vom Volk verworfen wurde (vgl. Bundesrat 2001 und 2003) - dazu beitrugen, dass die verstärkten Bemühungen im Lehrstellenmarketing positiv wahrgenommen und breit unterstützt wurden. Auf der Gegenseite zeigt das Fallbeispiel der finanziellen Anreize im Kanton Freiburg, dass die große Skepsis gegenüber der Maßnahme seitens der vollziehenden Behörden, die seit der Einführung der Maßnahme neu besetzt und reorganisiert worden waren, einen starken Unwillen beim Vollzug zur Folge hatte.

\section{Prozessverlauf und Akteurverhalten}

Auf der Ebene der politischen Prozesse und des Auftretens der beteiligten Akteure erweisen sich im Vergleich der untersuchten Projekte zwei Aspekte als bedeutsam für den Erfolg einer Maßnahme: die sinnvolle Integration durch Mitsprache der betroffenen Interessen bei der Ausgestaltung der Maßnahme und Eignung und Engagement der zuständigen Personen.

Die Bedeutung der breiten Mitsprache aller betroffenen Interessen bei der Ausgestaltung einer Maßnahme zeigt sich am Beispiel der Ausbildungsforen im Kanton Zürich. Foren sind dann erfolgreich, wenn sie alle wichtigen Institutionen an der Schnittstelle Volksschule-Berufsbildung des jeweiligen Bezirks versammeln können. Die Teilnahme der Wirtschaftsverbände und öffentlichen Arbeitgeber und Arbeitgeberinnen mit starken und engagierten Vertretungen erweist sich dabei als besonders entscheidend. Wenn nicht eine von den Verbänden akzeptierte und delegierte Vertretung im Forum teilnimmt, kann sich diese schlechte Einbindung auf die Akzeptanz des Forums und dessen Aktivitäten auswirken. Die Bedeutung der Mitsprache der verschiedenen Interessen zeigt sich auch beim einzigen Fall der Untersuchung, der von einem Verband getragen wird: Beim Lehrstellenmarketing der Bäcker-Konditoren-Confiserie-Branche hatte die wiederholte Berücksichtigung sprachregionaler Sensibilitäten eine große psychologische Bedeutung, da 
damit von vornherein ein Einbezug der jeweiligen Erwartungen signalisiert wurde. Gerade bei Marketingmaßnahmen und Kampagnen sind kulturelle Unterschiede von zentraler Bedeutung. Auch im Falle des basellandschaftlichen Lehrstellenförderers trug der Einsatz einer Arbeitsgruppe für die Projektentwicklung zur breiten Akzeptanz der Maßnahme bei. Wiederum als gegenteiliges Beispiel kann das Neuenburger Projekt zur Illustration herbeigezogen werden. Die fehlende Mitsprache des kantonalen Berufsbildungsamts bei der Ausgestaltung der Lehrstellenakquisition durch die dominante Berufsfachschule erhöhte die Skepsis gegenüber der Maßnahme.

Ein zweiter Faktor betrifft die Eignung und Akzeptanz der verantwortlichen Personen. Es zeigt sich am Beispiel der Zürcher Ausbildungsforen, dass ein Erfolg der Marketing-Tätigkeiten stark vom Engagement der einzelnen Leitungspersonen abhängt. Den Leitungspersonen kommt für den erfolgreichen Aufbau und für die gute Führung eines Forums eine besondere Bedeutung zu. Sie sind es, die Mitglieder mobilisieren, Aktivitäten planen, und damit das Forum in der Region verankern. Da die Foren weniger Organisationen als lockere Zusammenschlüsse sind, die vom Engagement der Beteiligten leben, braucht es Leute, die eine Vernetzung anstreben und es schaffen, genug andere Personen und Institutionen zur Mithilfe überzeugen zu können, ohne dabei selbst eine zu dominante Stellung einzunehmen. Wie wichtig die Wahl geeigneter Persönlichkeiten für den Erfolg einer Maßnahme ist, zeigen auch die beiden untersuchten Lehrstellenförderer im Kanton Basel-Landschaft und im Kanton Neuenburg. Die große allgemeine Akzeptanz des basellandschaftlichen Lehrstellenförderers, seine Schlüsselposition in der Berufsbildung (Vermittler Wirtschaft und Kanton, Ansprechperson für alle) und seine Bekanntheit im gesamten Kanton ist auf verschiedene Faktoren zurückzuführen. Vorab sind das überdurchschnittlich hohe persönliche Engagement des Lehrstellenförderers und seine Freude an der Tätigkeit zu nennen. Daneben spielen jedoch seine große fachliche Kompetenz und der wirtschaftsnahe berufliche Hintergrund eine wichtige Rolle. Anders der Neuenburger Lehrstellenförderer, der mit einem schulischen Hintergrund von der Wirtschaft nicht akzeptiert wurde. Die entsprechenden kulturellen Unterschiede führten in Kombination mit dem grundsätzlichen Systemwechsel, den er zu vermitteln hatte, zu mangelndem Wohlwollen seitens der angesprochenen Unternehmen, was sich in einer lediglich geringen Zahl neu geschaffener Lehrstellen äußerte.

\section{Inhaltliche Ausgestaltung der Maßnahmen}

Auf inhaltlicher Ebene der gewählten Strategie und der konkreten Maßnahmen finden sich ebenfalls verschiedene Faktoren, die in ihrer Kombination einen Beitrag zum grundsätzlichen Gelingen des Projekts beigetragen haben. Es sind dies die Einbettung einer Maßnahme in eine Gesamtstrategie, das Abstützen auf Problemanalysen und entsprechenden Zielformulierungen sowie die Kombination unterschiedlich verbindlicher Maßnahmen. 
Eine Erkenntnis des Fallstudienvergleichs liegt darin, dass sich unterschiedliche Trägerschaften als unterschiedlich geeignet für verschiedene Formen des Lehrstellenmarketings erweisen. So zeigt sich, dass nicht-professionelle Trägerschaften, wie wir sie bei den Ausbildungsforen finden, erfolgreich sind bei Maßnahmen, die eine eher tiefe Verbindlichkeit bei der Zielgruppe aufweisen. Diese Trägerschaften eignen sich für die Integration verschiedener Akteure, erreichen aber bei der Umsetzung komplexerer Projekte ihre Grenzen. Für die Realisierung organisatorisch anspruchsvoller Maßnahmenpakete erweisen sich dagegen professionelle und mit genügend Ressourcen ausgestattete Verwaltungsstrukturen als besonders wichtig. Dies zeigt sich sowohl beim Zentralschweizer Projekt sowie beim Lehrstellenmarketing der Bäcker-Konditoren-Confiseur-Branche. Schließlich erweisen sich wirtschaftsnahe Trägerschaften als besonders geeignet für Maßnahmen, die den direkten Kontakt mit potenziellen Ausbildnern beinhalten. Hervorragendes Beispiel hierfür ist der basellandschaftliche Lehrstellenförderer, der der kantonalen Wirtschaftskammer angegliedert ist. Der Zusammenhang zeigt sich aber ebenso beim Verein Berufsbildung Zentralschweiz als Träger der größten Innerschweizer Berufsbildungsmesse wie auch in negativer Form beim tendenziellen Scheitern des Neuenburger Lehrstellenförderers, der eben nicht einen privatwirtschaftlichen, sondern einen pädagogischen Hintergrund hatte.

Als ein starker erfolgsfördernder Faktor erweist sich generell die Einbettung einer Marketingmaßnahme in ein umfassendes Gesamtkonzept. Die einheitliche Ausrichtung verschiedener Instrumente auf gemeinsame übergeordnete Zielsetzungen hat gleich mehrere Vorteile für die Umsetzung der Maßnahmen, wie am Beispiel des Zentralschweizer Lehrstellenmarketings illustriert werden kann. Erstens ermöglichte hier der klare Rahmen eine Lernfähigkeit und Flexibilität des Fachprojekts, 'Kommunikation', was die Ergänzung mit dem Schwerpunkt Lehrstellenmarketing ab 2003 ermöglichte. Um die übergeordneten Zielsetzungen zu verfolgen, war angesichts der veränderten Ausgangslage eine Adaption notwendig. Zweitens ermöglichte die Integration in das Masterprojekt einzelne Synergieeffekte über die Fachprojekt-Grenzen hinweg. Drittens konnte für das Lehrstellenmarketing im engeren Sinne ab 2003 auf große Vorarbeiten aufgebaut werden, die im Rahmen des vorgängigen Fachprojektverlaufs geleistet worden waren. Die vorangegangenen Aktivitäten hatten einen bedeutenden Sensibilisierungseffekt, der zur Akzeptanz der nachfolgenden Marketingkampagne und zur Empfänglichkeit der vermittelten Botschaften wesentlich beitrug. Auch das Marketing der Bäcker-Konditoren-Confiseur-Branche ist Teil eines Gesamtkonzepts, welches auf verschiedenen Ebenen ansetzt und sowohl Synergieeffekte erzielt als auch auf gewonnene Erfahrungen aufbauen kann.

In engem Zusammenhang mit dem Vorhandensein eines Rahmen gebenden Gesamtkonzepts stehen zwei weitere Elemente, das Abstützen auf eine Problemanalyse und die Kombination verschiedener Maßnahmen. Dies zeigt sich wiederum beim Lehrstellenmarketing der Bäcker-Konditoren-Confiseur-Branche. Für die Erarbeitung des Gesamtkonzeptes war eine fundierte Problema- 
nalyse unabdingbar, da erst auf dieser Grundlage eine angemessene Zielformulierung möglich war. Der finanzielle Anreiz im Kanton Freiburg wurde dagegen konzipiert, ohne sich auf eine Situationsanalyse zu stützen. Die fehlende Legitimation durch Vorabklärungen trug zur schlechten Akzeptanz der Maßnahme wesentlich bei. Die Definition konkreter Ziele ist zentral für die Wahl der geeigneten Maßnahmen, um die identifizierten Probleme effektiv anzugehen. Während Einzelmaßnahmen zwar kurzfristige Erfolge beschieden sein können, ist es für die Nachhaltigkeit von Marketingmaßnahmen notwendig, Synergien zu schaffen, die über kurzfristige Erfolge hinaus tragfähig sind und sie auf eine langfristige Perspektive ausrichten. Die Bedeutung der Kombination unterschiedlicher Instrumente zeigt sich auch beim Zentralschweizer Lehrstellenmarketing. Das Anschreiben potenzieller Ausbildungsbetriebe zeitigte vor allem dank Nachgreifaktionen Resultate. Die schriftliche Kontaktaufnahme war zwar unumgänglich für die angestrebte Breitenwirkung, in der Mehrheit der Fälle aber vertiefte erst der persönliche Kontakt das geweckte Interesse und führte zum erwünschten Verhalten der Adressaten, d.h. zur Schaffung neuer Lehrstellen.

\section{Institutionelle und organisatorische Faktoren}

Neben der Prozess- und Kontextebene und der inhaltlichen Ausgestaltung der Maßnahmen spielen schließlich auch organisatorische und institutionelle Aspekte eine zentrale Rolle für den Erfolg einer Marketingmaßnahme. Dies betrifft die Bereitstellung der notwendigen fachlichen und personellen Ressourcen, die Koordinationsfähigkeit der Projektleitung und die Gewährleistung der notwendigen Handlungsspielräume der Projektverantwortlichen bei der Umsetzung.

Erstens zeigt sich im Fallvergleich die nicht zu unterschätzende Bedeutung professionellen Fachwissens als auch personeller Ressourcen für die Wahrnehmung von Marketingmaßnahmen im Berufsbildungsbereich. Diese Bedingung trifft sowohl für die Verwaltung als auch für verbandliche Trägerschaften zu. Alle untersuchten Projekte stellten hohe fachliche Anforderungen an die konzipierenden und durchführenden Stellen. Erfolg stellte sich dort ein, wo die Bedingungen gegeben waren, diese Anforderungen zu erfüllen, d.h. wo die notwendige Expertise zur Verfügung stand. Dies ist beim Zentralschweizer Projekt, beim basellandschaftlichen Lehrstellenförderer sowie beim Lehrstellenmarketing der Bäcker-Konditoren-Confiseur-Branche der Fall, in denen fachlich ausgewiesenes Personal mit der Konzipierung und Umsetzung der jeweiligen Maßnahmen betraut war und dazu auch die entsprechenden personellen Ressourcen zur Verfügung standen.

Zweitens zeigt sich nicht allein bei den komplexen Projekten mit breitem Maßnahmenmix, sondern auch bei den Projekten, die sich auf ein bestimmtes Instrument konzentrieren, dass erfolgreiches Lehrstellenmarketing hohe Koordinationsanforderungen aufweist. Die Zentralschweizer Fallstudie illustriert, wie das gemeinsame Vorgehen von sechs Kantonen mit einer 
ausgesprochenen Zentralisierung der Arbeiten große Skaleneffekte bewirkte. Es war auch zu einem gewissen Grade der zentralisierten Organisationsform und der dadurch erzielten Aufwandsoptimierung im Fachprojekt 'Kommunikation' zu verdanken, dass 2003 noch genügend Ressourcen für die Neuausrichtung auf das Lehrstellenmarketing zur Verfügung standen. Auch beim Lehrstellenmarketing der Bäcker-Konditoren-Confiseur-Branche eröffnete die zentralisierte Vorgehensweise neue Handlungsspielräume, die im Sinne der definierten Projektziele für eine strategische Ergänzung der Maßnahmen durch eine Marketingkampagne genutzt wurden.

Schliesslich ist auf die Bedeutung der operativen Autonomie bei der Umsetzung der Maßnahmen hinzuweisen. Die Trennung der politisch-strategischen Sphäre von der eigentlichen Leistungserbringerseite erweist sich gleich bei einer Mehrzahl der untersuchten Fälle als bedeutender Erfolgsfaktor. Der Erfolg der Zürcher Ausbildungsforen ist zu einem gewissen Grad auf ihre jeweilige Autonomie bei der Erfüllung ihrer Leistungsvereinbarungen mit dem Kanton zurückzuführen. Diese Autonomie ermöglicht die flexible Ausrichtung der Tätigkeiten an den regionalen Bedürfnissen und die Wahl der geeigneten Mittel. Ebenso spielte beim Zentralschweizer Masterprojekt die Autonomie, die den Fachprojektverantwortlichen gewährt wurde, eine wichtige Rolle. Durch die klare Aufgabenzuteilung wurden Handlungsspielräume geschaffen und gesichert, die den Fachprojektverantwortlichen nicht nur eine effiziente operative Arbeit, sondern auch eine strategische Gestaltungsfreiheit ermöglichten, ohne die es beispielsweise kaum zur Initiative für die untersuchte Lehrstellenmarketingkampagne gekommen wäre. Ausgesprochen große Autonomie genießt der basellandschaftliche Lehrstellenförderer, was ihm ermöglicht, seine Arbeit bedürfnisgerecht zu entfalten - in diesem Fall ein wesentlicher Erfolgsfaktor. Schließlich spielt die strikte Aufgabenteilung und die Definition klarer Handlungsspielräume für den Fachexperten bei der Geschäftsstelle der beiden Verbände der Bäcker-Konditoren-Confiseur-Branche eine zentrale Rolle für die Erarbeitung eines professionellen Konzepts zur Nachwuchssicherung.

\section{Schlussfolgerungen}

Der Vergleich von sechs unterschiedlichen Projekten im Bereich des Lehrstellenmarketings führt zu Erkenntnissen, die über das Rahmen gebende Schweizer Berufsbildungssystem hinaus für die Konzipierung und Durchführung solcher Projekte nutzbar gemacht werden können. Im Folgenden werden in diesem Sinne Schlussfolgerungen aus der Untersuchung gezogen, die eine konkrete politisch-praktische Ausrichtung haben, und auf die langfristige Sicherung der Vorteile des dualen Systems zielen. Dabei wird in einem ersten Schritt auf die generelle Ausrichtung und anschließend konkreter auf erfolgsfördernde Projekteigenschaften eingegangen. 


\section{Generelle Ausrichtung von Lehrstellenmarketing}

Es bestätigt sich, dass Betriebe und Verbände für die Lösung des Lehrstellenproblems entscheidende Akteure sind. Im Fallstudienvergleich hat sich gezeigt, dass wirtschaftsnahe Trägerschaften und Akteure viel zum Erfolg von Marketingprojekten beitragen. Es ist deshalb sinnvoll, wenn vermehrt Projekte durchgeführt werden, welche die Angebotsseite (Betriebe) ansprechen. Dabei sind auch Projekte von Verbänden der Arbeitswelt erstrebenswert. Aus den gleichen Gründen ist die Marketingform 'Interventionen', namentlich die Lehrstellenakquisition und Beratung durch Betriebsbesuche sinnvoll.

\section{Erfolgversprechende Projekteigenschaften}

Um die Nachhaltigkeit sowie die Wirksamkeit von Lehrstellenmarketing sicherstellen zu können, erweisen sich vor allem Projekte mit folgenden Eigenschaften als erfolgreich:

Projekte, die auf bestehende und gut funktionierende Strukturen und Institutionen aufbauen, können langfristig weitergeführt werden. Zudem verfügen diese Institutionen oft auch über genügend Fachwissen, personelle Ressourcen und professionelle Strukturen.

Damit Marketingmaßnahmen zielgerichtet ein- und umgesetzt werden können, sollten sie in ein umfassendes Gesamtkonzept für die Berufsbildung eingebettet sein. Damit eine angemessene Zielformulierung sowohl für das Gesamtkonzept wie auch für einzelne Maßnahmen überhaupt möglich ist, muss vorgängig eine Problemanalyse erstellt werden. Erst aufgrund dieser Analyse können konkrete Ziele und darauf aufbauend die geeigneten Maßnahmen ausgewählt werden.

Eine Evaluation der Marketingprojekte ist nur möglich, wenn eine klar definierte Zielsetzung und daraus abgeleitete Maßnahmen mit evaluierbaren Elementen vorhanden sind. Die Evaluation von Marketingvorhaben ist unabdingbar, damit nötige Korrekturen und Verbesserungen angebracht werden können.

Bei der Ausgestaltung einer Marketingmaßnahme sollten alle betroffenen Interessen integriert werden, damit die Maßnahme bei der Umsetzung über genügend Akzeptanz verfügt. Damit die Mitsprache der Akteure gewährleistet werden kann, muss für die Konzipierungsphase der Projekte genügend Zeit einberechnet werden. Eine gute Planung ist unabdingbar.

Die Marketingvorhaben sollten über eine geeignete Trägerschaft verfügen. Für organisatorisch und inhaltlich anspruchsvolle Maßnahmen braucht es professionelle und mit genügend Ressourcen ausgestattete Verwaltungs- oder Verbandsstrukturen. Maßnahmen mit einer tiefen rechtlichen Verbindlichkeit für die Zielgruppen können auch von nicht professionellen Organisationen getragen werden. Wirtschaftsnahe Trägerschaften sind für den Marketingtyp der ,Interventionen', der den direkten Kontakt mit (potenziellen) Lehrbetrieben beinhaltet, besonders geeignet. 
Eine Kombination von verschiedenen Maßnahmen ist anzustreben. So sollten Informationskampagnen für Betriebe mit Akquisitionselementen begleitet werden. Der Effekt von Informations- und Sensibilisierungskampagnen für die Nachfrageseite kann deutlich vergrößert werden, wenn zur gleichen Thematik und in der gleichen Zeitspanne Projekte durchgeführt werden. Neue Maßnahmen, Modelle oder Projekte können umgekehrt mit Informationskampagnen unterstützt werden.

Die Trennung der strategisch-politischen Seite und der eigentlichen Leistungserbringerseite hat sich bei vielen Marketingvorhaben bewährt. So haben die Projektbeauftragten genügend operative Autonomie bei der Umsetzung der Maßnahme und können bedürfnisgerecht arbeiten. Eine zentralisierte Arbeits- und Vorgehensweise gewährleistet eine gute Koordination, welche für erfolgreiches Lehrstellenmarketing unabdingbar ist.

\section{Literaturverzeichnis}

Borham, Nick. 2002. Work Process Knowledge, Curriculum Control, and the WorkBased Route to Vocational Qualifications. British Journal of Educational Studies 50(2): 225-327.

BIBB (Bundesinstitut für Berufsbildung). 2004. Berufsbildungsfachleute sind optimistisch: Duales Berufsausbildungssystem hat auch in Zukunft Bestand! Pressemitteilung 40/2004. Bonn, 10.11.2004 (http://www.bibb.de/de/15959.htm, abgerufen am 11. Januar 2005).

Bundesbeschluss. 1999. Bundesbeschluss über Maßnahmen zur Verbesserung des Lehrstellenangebotes und zur Entwicklung der Berufsbildung (Lehrstellenbeschluss II) vom 18. Juni 1999. Bern: Schweizerische Eidgenossenschaft.

Bundesrat. 2000. Botschaft zu einem neuen Bundesgesetz über die Berufsbildung (Berufsbildungsgesetz, BBG) vom 6. September 2000 (BBl 2000 5686). Bern: Schweizerische Eidgenossenschaft.

2001. Botschaft über die Volksinitiative "für ein ausreichendes Berufsbildungsangebot (Lehrstellen-Initiative)" vom 25. Oktober 2000 (BBl 2001 97). Bern: Schweizerische Eidgenossenschaft.

—. 2003. Bundesratsbeschluss über das Ergebnis der Volksabstimmung vom 18. Mai 2003 vom 10. Juli 2003 (Armee XXI; Bevölkerungsschutz und Zivilschutz; Initiative "Ja zu fairen Mieten"; "Sonntags-Initiative"; "Gesundheitsinitiative”; Initiative "Gleiche Rechte für Behinderte"; Initiative "Strom ohne Atom"; Initiative "MoratoriumPlus"; "Lehrstellen-Initiative") ( BBl 2003 5164). Bern: Schweizerische Eidgenossenschaft.

Deißinger, Thomas, und Silke Hellwig. 2004. Initiatives and Strategies to Secure Training Opportunities in the German Vocational Education and Training System. Journal of Adult and Continuing Education 10(2): 160-174.

DBK (Deutschschweizerische Berufsbildungsämter-Konferenz). 2004. Berufliche Bildung in der Schweiz. (http://www.dbk.ch/broschuere/ch3/ch3to.htm; abgerufen am 14.06.04).

Freitag, Markus, und Marc Bühlmann. 2003. Die Bildungsfinanzen der Schweizer Kantone. Der Einfluss sozioökonomischer Bedingungen, organisierter Interessen und politischer Institutionen auf die Bildungsausgaben im kantonalen Vergleich. Swiss Political Science Review 9(1): 139-168. 
Gertsch, Marianne, und Hans-Peter Hotz. 1999. Der Lehrstellenbeschluss. Evaluation. Studie zum Lehrstellenmarketing. Bern: Koordinationsstelle für Weiterbildung (KWB) der Universität Bern.

Labarca, Guillermo. 1998. Education in Basic Skills and Training for Productive Work. International Review of Education 44(5/6): 413-439.

Lee, Lung-Sheung. 1994. Vocational-Technical Education Reform in Germany, Netherlands, France and UK and Their Implication to Taiwan. Paper presented at the annual meeting of the American Vocational Assocation, Dallas (TX), USA.

Müller, Walter, und Yossi Shavit. 1998. Bildung und Beruf im institutionellen Kontext. Eine vergleichende Studie in 13 Ländern. Zeitschrift für Erziehungswissenschaft 1(4): 501-534.

Sager, Fritz, Martina Schläpfer, und Olivier Giraud. 2004. Vertiefungsstudie Lehrstellenmarketing. Quantitative und qualitative Untersuchung der spezifischen Lehrstellenmarketingprojekte der Kantone und des Bundes im LSB2. Bern: Bundesamt für Berufsbildung und Technologie.

Schucan, Luzi. 2002. Berufliche Nachwuchsförderung. Gesamtkonzept und Maßnahmen zur weiteren Entwicklung im Kanton Zürich. Zürich: Bildungsentwicklung, Mittelschulund Berufsbildungsamt des Kantons Zürich; IleB - Institut für Lehrerbildung und Berufspädagogik; Hochschulamt des Kantons Zürich.

Utiger, Urs. 2003. Berufsbildung 1985-2003 im Kanton Zürich: Wenig Lehrstellen in attraktiven Dienstleistungsbranchen. Zürich: Statistisches Amt des Kantons Zürich (statistik.info 22/2003).

Wolter, Stefan C., und Jürg Schweri. 2004. Ökonomische Aspekte der Organisation einer Berufslehre. Zeitschrift für Berufs- und Wirtschaftspädagogik 100(1): 13-25.

Stefan C., Wolter, und Jürg Schweri. 2002. The Cost and Benefit of Apprenticeship Training: The Swiss Case. Applied Economics Quarterly (Konjunkturpolitik) 48(3-4): 347-367.

Wolter, Stefan C., Samuel Mühlemann, und Jürg Schweri. 2003. Why Some Firms Train Apprentices and Many Other Do Not. IZA Discussion Paper No. 916. Bonn: IZA.

Stefan C., Wolter, Andrea Nagel-Drdla, und Roland Waibel. 2001. Bildungsfinanzierung zwischen Markt und Staat. Chur/Zürich: Rüegger.

\section{Der Autor}

Fritz Sager ist Assistenzprofessor für Politikanalyse und -evaluation am Institut für Politikwissenschaft der Universität Bern. Er ist Autor mehrerer politikwissenschaftlicher Publikationen sowie einer Vielzahl von Forschungsberichten und Expertisen im Auftrag der schweizerischen Bundesverwaltung und weiterer Auftraggeber. Seine inhaltlichen Schwerpunkte sind neben der Bildungspolitik die Gesundheits- und Sozialpolitik, die raumwirksamen Politikfelder Verkehr, Raum und Umwelt sowie die Außenpolitik.

Contact address: Fritz Sager, Institute of Political Science University of Bern, Unitobler, Lerchenweg 36, CH-3000 Bern 9, Switzerland, E-mail: sager@ipw.unibe.ch 